mutation rate. This indirect selective force is considerably weakened by genetic recombination, a process that breaks apart linked genes - and a factor not included by Kamp et al. in their model. But recombination is substantial in many viruses, and its effect should probably be considered explicitly in modelling the evolution of viral mutation rates.

Despite these shortcomings, the paper of Kamp et al. ${ }^{2}$ is clearly an important conceptual development in the study of mutation-rate evolution in viruses. Moreover, developing a fuller understanding of the evolutionary causes and consequences of viral mutation rates is worthwhile from both basic and applied perspectives. Drugs that increase genomic mutation rates can kill off viral populations by causing them to exceed their error threshold ${ }^{9,10}$. A quantitative theory that can predict how close to the error threshold a given viral population is - without the need to estimate its mutation rate directly — might have real therapeutic value.

Sebastian Bonhoeffer is in the Department of

Environmental Science, ETH Zürich, CH-8092

Zürich, Switzerland.

e-mail:bonhoeffer@eco.umnw.ethz.ch

Paul Sniegowski is in the Department of Biology,

University of Pennsylvania, Philadelphia,

Pennsylvania 19104, USA

e-mail:paulsnie@sas.upenn.edu

1. Drake, J. W., Charlesworth, B., Charlesworth, D. \& Crow, J. F. Genetics 148, 1667-1686 (1998).

2. Kamp, C., Wilke, C. O., Adami, C. \& Bornholdt, S. Complexity

(in the press); Preprint cond-mat/0209613 (2002),

http://arXiv.org

3. Eigen, M. Naturwissenschaften 58, 465-523 (1971).

4. Kimura, M. Genet. Res. 9, 23-34 (1967).

5. Leigh, E. G. Genetics 73, 1-18 (1973)

6. Ishii, K., Matsuda, H., Iwasa, Y. \& Sasaki, A. Genetics 121, 163-174 (1989).

Nowak, M. A. Nature 347, 522 (1990).

8. Sniegowski, P. D., Gerrish, P. J., Johnson, T. \& Shaver, A. BioEssays 22, 1057-1066 (2000)

9. Loeb, L. A. et al. Proc. Natl Acad. Sci. USA 96, 1492-1497 (1999).

10. Crotty, S., Cameron, C. E. \& Andino, R. Proc. Natl Acad. Sci. USA 98, 6895-6900 (2001).

\title{
Fixation with regulation
}

\section{J. Allan Downie and Martin Parniske}

\section{A gene has been isolated that controls the number of symbiotic nitrogen-} fixing nodules in legumes. Its similarity to a well-characterized regulatory gene in Arabidopsis provides clues about its action.

eguminous plants produce root nodules, within which symbiotic bacteria capture atmospheric $\mathrm{N}_{2}$ and convert it into nitrogen that can be used by the plant. But this process is energetically expensive and so legumes strictly control the numbers of nodules they form. Papers by Krusell et al. ${ }^{1}$ and Nishimura et al. ${ }^{2}$ (pages 422 and 426 of this issue), and by Searle et al. ${ }^{3}$ in Science, describe the characterization of a regulatory gene that normally limits nodule numbers, and that when mutated increases nodulation. Control of nodule development is of interest in its own right, but this work may also have agricultural applications.

Soybean and pea mutants with enhanced nodulation have been known for about 20 years ${ }^{4}$, but their complex genomes have hampered attempts to clone the genes responsible. Similar mutants ${ }^{5,6}$ were recently identified in Lotus japonicus, a legume with a relatively small genome. These mutants have hypernodulation and aberrant roots, hence their designation as har mutants. The numbers of both nodules and lateral roots are increased in these L. japonicus and soybean mutants, indicating that normal legumes possess a common regulatory mechanism that limits the numbers of root and nodule growing points, or meristems.

Grafting experiments showed that HAR1 control of nodule and lateral-root number in $L$. japonicus depends on the shoots rather than the roots (Fig. 1, overleaf), a characteristic that had been previously observed with soybean hypernodulation mutants ${ }^{4}$. So plants with har1-mutant shoots grafted onto wild-type roots had increased root nodulation. In contrast, the reciprocal grafted plants (mutant roots with wild-type shoots) had normal roots and nodules. After positioning HAR 1 on a physical map of the L. japonicus genome, two groups ${ }^{1,2}$ cloned the gene. They went on to identify mutations in the equivalent genes in pea ${ }^{1}$ and soybean ${ }^{2}$ hypernodulation plants, which showed a similar shoot control of nodulation ${ }^{3}$. Independently, following about 15 years of work ${ }^{3}$, the equivalent gene controlling nodulation in soybean was isolated and was called NARK (nodule autoregulation receptor kinase). It is clear that HAR1 and NARK are the same genes from different species.

HAR 1 and NARK encode a type of receptor protein that is abundant in plants ${ }^{7}$ and has three components: an extracellular domain of leucine-rich repeats, a membrane-spanning domain, and an intracellular protein kinase domain (Fig. 2). This structure is compatible with the receptor's function being perception of a ligand outside the cell, followed by internal signal transduction through protein phosphorylation by the kinase domain. Mutant genes sequenced from the three species had

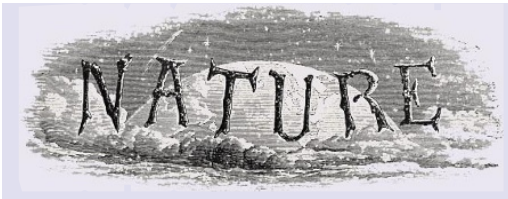

100 YEARS AGO

We have received from Mssrs. J. W. Gray and Son a pamphlet on scientific protection against lightning, written by Mr. A. Hands. The writer gives a careful explanation of the principles which must be observed in erecting lightning conductors; as the pamphlet is written in non-technical language, it is to be hoped it may be the means of disseminating information amongst the public, since there are few subjects on which more ignorance and superstition exist. The importance of careful protection may be gathered from the fact that Mr. Hands estimates the damage caused annually by lightning in this country alone at from 50,000 l. to 100,000 l.

ALSO

The Liverpool correspondent of the Central News states that the Nobel prize of 3,000 l. for researches in connection with malaria will be a personal one to Major Ross, principal of the Liverpool School of Tropical Medicine. According to the Stockholm correspondent of the Daily Chronicle, the prize for medicine will be awarded to Prof. Finsen, the Danish discoverer of the treatment by red light for lupus, and the prize for physics to Prof. S. A. Arrhenius. From Nature 27 November 1902.

\section{YEARS AGO}

On November 4 at 16h. 58m. 20s. G.M.T., an earthquake occurred with epicentre... near the east coast of Kamchatka. It was recorded strongly at seismological observatories throughout the world and had a magnitude of $81 / 3$ on the GutenbergRichter logarithmic scale... Faulting probably took place in the sea bed near the epicentre since a great tsunami or seismic sea wave resulted, and spread throughout the Pacific Ocean. It arrived at the coast of northern Japan about 20h. G.M.T. on November 4. The Hawaiian warning system was used and the coastlines of several islands, including the Oahu coast, were evacuated in anticipation of the wave. When the wave arrived at Hawaii itself, it is reported to have been several feet high... Waves from one to three feet high arrived at the Whangarei beaches in the north of New Zealand about 7 p.m. local time on November 5. When these waves arrived at Wellington, they were 6-8 in. high... Immediately following the main shock, there were more than a hundred aftershocks. Further news is awaited.

From Nature 29 November 1952. 


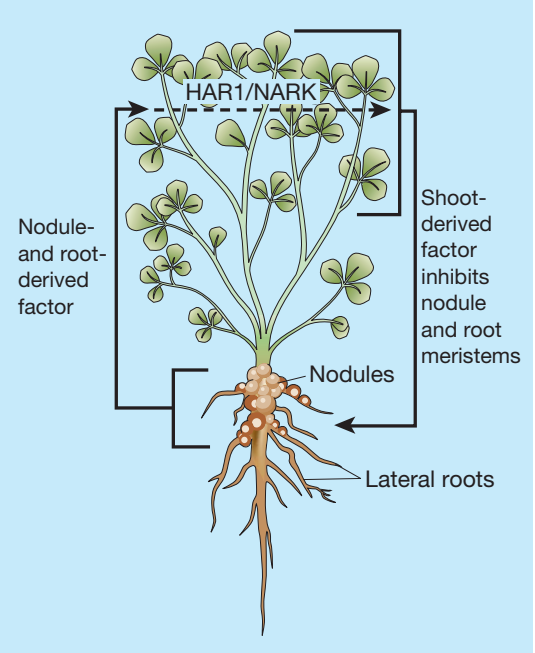

Figure 1 Control of nodule number in legumes. One proposal ${ }^{4}$ is that a factor produced by nodule and root meristems is detected in shoots, resulting in the production of a second factor that suppresses the development of new nodules. In line with this model, mutation of the HAR1/NARK gene increases the numbers of nodules and lateral-root meristems ${ }^{1-3}$. So HAR1/NARK protein is required for the production of the shoot-derived inhibitor, possibly through its involvement in perception of a nodule- and root-derived signal.

alterations in the kinase domain, suggesting that phosphorylation by HAR1/NARK protein is essential for signalling. The signal perceived by HAR1/NARK is unknown, as is the kind of inhibitor that suppresses further nodulation.

Comparison with the model plant Arabidopsis provides insight into how HAR1/ NARK might function, even though Arabidopsis does not form nodules. The Arabidopsis genome is predicted to contain 216 leucinerich-repeat, receptor-like kinases ${ }^{7}$. They are believed to perceive extracellular signals such as bacterial flagellin, the wounding signal systemin, and the plant hormones brassinolide and phytosulphokine. A real surprise from the new work ${ }^{1-3}$ is that the legume HAR1/NARK is more similar in sequence to Arabidopsis CLAVATA1 than to any other Arabidopsis protein, implying that, like CLAVATA1, it functions as a receptor kinase. Together with CLAVATA2, CLAVATA1 forms a complex that detects the signal peptide CLAVATA3 (Fig. 2). The CLAVATA genes are involved in regulating cell fate in the shoot apical meristems ${ }^{8}$, clavatal mutants having an enlarged meristematic zone that leads to fasciation (contiguous parts growing into one)

CLAVATA1 and HAR1/NARK appear to have similar functions, because mutation of the corresponding genes results in increased meristematic activity (Fig. 2), either in shoot apical meristems (clavata1) or in root and nodule meristems (harl/nark). However, they must have different roles, because the roots of clavata1 mutants are unaffected, as are the apical meristems of nark mutants ${ }^{3}$. Moreover, their patterns of expression are different. CLAVATA1 is exclusively expressed in apical meristems ${ }^{8}$, whereas HAR/NARK seem to be expressed in most tissues except apical meristems ${ }^{2,3}$. It will be interesting to see how far the different functions of HAR1/NARK and CLAVATA1 can be attributed to their expression patterns.

Grafting and other experiments with soybean hypernodulation mutants led to a model of how legumes might regulate nodule number ${ }^{4}$. This postulates that a signal moves from the root to the shoot, and that increased root nodulation is detected in shoots as the root-derived signal increases. As a result, the shoots produce an inhibitor, which is translocated to the root, where it represses further nodule development. Based on the grafting experiments and the nature of HAR1/NARK, it seems that this protein could be involved in the perception of a root-made signal in the shoot (Fig. 1). An unexpected result is that all three groups ${ }^{1-3}$ detected HAR1/NARK messenger RNA in roots, implying that the protein is being made there. But although the gene is transcribed in roots, the grafting experiments show that the root-expressed gene alone cannot function in nodule repression. So some additional factor in the aerial part of the plant may be required for HAR1/NARK to act.

There is a second shoot-controlled hypernodulation gene in pea that, when mutated, can lead to shoot fasciation ${ }^{9}$. Given the similar fasciation seen with the clavata mutants, there may be some functional overlap of the CLAVATA and HAR1/NARK pathways. It seems likely that the product of this second gene in pea could be part of the same signalling pathway as HAR1/NARK. Identification of the gene will be required to see if its product can interact directly with HAR1/NARK (Fig. 2) and what relationship there is between the HAR1/NARK and CLAVATA pathways. Genes with high sequence similarity to CLAVATA1 have been identified in soybean ${ }^{10}$, and so HAR1/NARK may have arisen as a duplication of an ancestral CLAVATA1- like gene, with a subsequent divergence of functions.

Finally, the results of Krusell et al. ${ }^{1}$, Nishimura et al. ${ }^{2}$ and Searle et al. ${ }^{3}$ have practical implications. One of the problems with most of the existing hypernodulation mutants is that the abnormal root and nodule development has severe effects on plant growth. However, Searle et al. ${ }^{3}$ describe one mutation in NARK that has less detrimental effects. A different L. japonicus gene encoding a transcriptional regulator that represses nodulation has also just been described ${ }^{11}$, but its relationship to the HAR1/NARK receptor pathway is not known. Now that

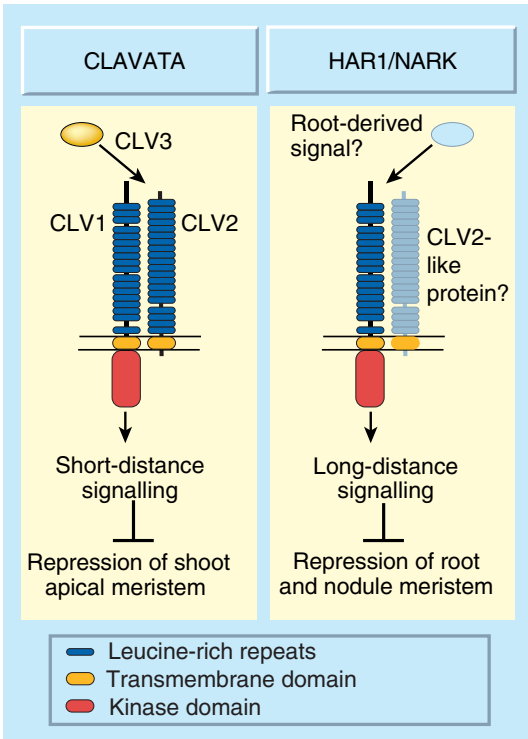

Figure 2 How HAR1/NARK might function in signalling and root nodulation. This scheme is based on the model ${ }^{8}$ for signalling by CLAVATA proteins in Arabidopsis, and on the characteristics of HAR1/NARK described by Krusell et al. ${ }^{1}$, Nishimura et al. ${ }^{2}$ and Searle et al. ${ }^{3}$. HAR1 is most similar to CLAVATA (CLV) 1, a receptor kinase containing leucine-rich repeats which interacts with CLV2, forming a complex that recognizes the signalling peptide CLV3. CLV2 contains leucine-rich repeat and transmembrane domains, but lacks a kinase domain. HAR1/NARK might interact with a CLV2-like protein, yet to be identified, to recognize a signal produced by root and nodule meristems.

key genes regulating nodulation have been isolated, it may be possible to identify plants carrying subtle mutations - these might allow increased nodulation, with consequent increases in nitrogen fixation, without affecting plant growth too badly. Such plants might show improved growth, and also leave residual nitrogen in the soil to increase the growth of subsequent crops.

J. Allan Downie is in the Department of Molecular Microbiology, and Martin Parniske is in the

Sainsbury Laboratory, John Innes Centre,

Colney Lane, Norwich NR4 7UH, UK.

e-mails:allan.downie@bbsrc.ac.uk

martin.parniske@sainsbury-laboratory.ac.uk

1. Krusell, L. et al. Nature 420, 422-426 (2002).

2. Nishimura, R. et al. Nature 420, 426-429 (2002).

3. Searle, I. R. et al. Science published online 31 October 2002 (doi: 10.1126/science.1077937).

4. Caetano-Anolles, G. \& Gresshoff, P. M. Annu. Rev. Microbiol. 45, 345-382 (1991)

5. Wopereis, J. et al. Plant J. 23, 97-114 (2000)

6. Kawaguchi, M. et al. Mol. Plant-Microbe Interact. 15, 17-26 (2002).

7. Shiu, S.-H. \& Bleecker, A. B. Proc. Natl Acad. Sci. USA 11, 10763-10768 (2001)

8. Clark, S. E. Nature Rev. Mol. Cell Biol. 2, 276-284 (2001).

9. Sagan, M. \& Duc, G. Symbiosis 20, 229-245 (1996).

10. Yamamoto, E., Karakaya, H. C. \& Knap, H. T. Biochim. Biophys. Acta 1491, 333-340 (2000).

11. Nishimura, R., Ohmori, M., Fujita, H. \& Kawaguchi, M. Proc. Natl Acad. Sci. USA 99, 15206-15210 (2002). 